\title{
Bulk Waves in the Infinite Electric-Magnetic-Elastic Plate with Mixed Boundary Conditions
}

\author{
Genquan Xie ${ }^{1 *}$, Xingpeng Song1, Xiaodong Xiao ${ }^{2}$ \\ ${ }^{1}$ Civil Engineering School, Hunan University of Science and Technology, Xiangtan, China \\ ${ }^{2}$ Experimental School Affiliated to Loudi No. 1 Middle School, Loudi, China \\ Email: *xiaoyuanyixiong@163.com
}

How to cite this paper: Xie, G.Q., Song, X.P. and Xiao, X.D. (2021) Bulk Waves in the Infinite Electric-Magnetic-Elastic Plate with Mixed Boundary Conditions. Journal of Electromagnetic Analysis and Applications, 13, 21-39.

https://doi.org/10.4236/jemaa.2021.132002

Received: February 17, 2021

Accepted: February 25, 2021

Published: February 28, 2021

Copyright (อ 2021 by author(s) and Scientific Research Publishing Inc. This work is licensed under the Creative Commons Attribution International License (CC BY 4.0).

http://creativecommons.org/licenses/by/4.0/

\begin{abstract}
A dynamic solution is presented for the propagation of waves in an electric-magneto-elastic plate composed of piezoelectric, piezomagnetic materials and elastic matrix. The electric-magneto-elastic plate is polarized along the thickness direction. The generalized displacements are expressed as the sum of the gradient of a scalar (dilatation wave) and the curl of a vector (shear wave). With the help of dynamic equilibrium equations and geometric equations, we can obtain dynamic equations of the dilatation wave and the shear wave. The conclusion that the types of the dilatation waves and the shear waves remain unchanged after being reflected by the boundary can be obtained through the analysis of these kinetic equations. The dispersion properties and phase velocity surface of the dilatation and shear wave can be obtained by solutions of dynamic equilibrium equations. Influences of the piezoelectric and piezomagnetic parameters on wave characteristics are discussed.
\end{abstract}

\section{Keywords}

Bulk Wave, Electric-Magneto-Elastic Plate, Dilatation Wave, Shear Wave, Dispersion Properties, Phase Velocity Surface

\section{Introduction}

The electric-magneto-elastic structures or materials have the capacity of converting energy from one form to the other (among mechanical, magnetic and electric energy) [1] [2], and show good application prospects in smart sensors, actuators, transducers and many other emerging devices. There is a strong need 
for theories or techniques that can predict the coupled response of these smart materials, as well as structures made up of them. Static and dynamic characteristics of plates as well as infinite cylinder have been studied in the literature. Piotr Cupiał [3] discussed a perturbation solution of the natural frequencies and mode shapes of a piezoelectric rectangular plate modeled as a three-dimensional body. Guoquan Nie et al. [4] instigated shear horizontal ( $\mathrm{SH}$ ) waves propagating in piezoelectric-piezomagnetic bilayer system with an imperfect interface. Hamdi Ezzin et al. [5] presented a dynamic solution for the propagation of harmonic waves in magneto-electro-elastic plates composed of piezoelectric $\mathrm{BaTiO}_{3}(\mathrm{~B})$ and magnetostrictive $\mathrm{CoFe}_{2} \mathrm{O}_{4}(\mathrm{~F})$ material. Xiao Guo et al. [6] dealt with effects of functionally graded interlayers on dispersion relations of shear horizontal waves in layered piezoelectric/piezomagnetic cylinders. J.G. Yu et al. [7] proposed a double orthogonal polynomial series approach to solving the wave propagation problem in a two-dimensional (2-D) structure. Yu Pang et al. [8] employed the stiffness matrix method and the transfer matrix method to investigated $\mathrm{SH}$ bulk/surface waves propagating in the corresponding infinite/semi-infinite piezoelectric (PE)/piezomagnetic (PM) and PM/PE periodically layered composites. Feng-Ming Li et al. [9] investigated the elastic wave propagation in phononic crystals with piezoelectric and piezomagnetic inclusions. Wei and $\mathrm{Li}$ [10] investigated the direction dependence of surface wave speed and the influence of electrically and magnetically short/open circuit conditions. Yu Pang et al. [11] analyzed the reflection and refraction of a plane wave incidence obliquely at the interface between piezoelectric and piezomagnetic media. M. Arfi [12] used nonlocal elasticity to analyze waves in a functionally graded magneto-electro-elastic nano-rod.

The above existing works of literature are mainly researches on surface waves in electric-magneto-elastic plates. Hence in the present study, characteristics of bulk wave in an electric-magneto-elastic plate have been studied, the physical of waves and the geometric dispersion of waves along the thickness of an infinite plate are simultaneously studied. The elastic displacement is expressed as the sum of a scalar gradient and a vector curl. Based on dynamic equilibrium equations and geometric equation, we can obtain dynamic equations of the dilatation wave and shear wave, and the dispersion properties and phase velocity surface of the dilatation and shear wave can be obtained by solutions of dynamic equilibrium equations. The main aim of the study is to evaluate the influence of piezoelectric, piezomagnetic and elastic parameters on characteristics of bulk wave in an electric-magneto-elastic plate.

\section{Formulation}

The electric-magneto-elastic material possess characteristic of the magnetic-electric-mechanical coupling. In this paper, only wave of magnetic potential and electric one and displacements are considered, so that the displacement vector $\boldsymbol{u}$, electric potential $\varphi$, and magnetic potential $\psi$ are chosen as basic 
variables. The basic equations are listed as follows.

The constitutive equations of the electric-magneto-elastic material are given by

$$
\begin{aligned}
& \sigma_{i j}=C_{i j k l} \varepsilon_{k l}-e_{i j n} E_{n}-q_{i j n} H_{n} \\
& D_{n}=e_{k l n} \varepsilon_{k l}+g_{m n} E_{m}+\alpha_{m n} H_{m} \\
& B_{n}=q_{k l n} \varepsilon_{k l}+\alpha_{m n} E_{m}+\mu_{m n} H_{m}
\end{aligned}
$$

where $\sigma_{i j}, \varepsilon_{k l}, D_{m}$ and $B_{m}$ are, respectively, stresses, strains, electric displacements and magnetic induction tensors, $C_{i j k l}$ are the elastic constants, $e_{i j n}$ the piezoelectric tensors, $g_{m n}$ the dielectric tensors, $\alpha_{m n}$ the magnetic-electric mutual inductance tensors, $q_{i j n}$ the piezomagnetic tensors, $\mu_{m n}$ the magnetic permeability tensors, all the subscripts $i, j, k, l, m, n=1,2,3$, two identical subscripts in an integral expression denote the sum of Einstein.

The generalized dynamic equilibrium equations absent of the mechanical body force are given by

$$
\begin{gathered}
\sigma_{i j, j}=\rho \ddot{u}_{i} \\
D_{n, n}=0 \\
B_{n, n}=0
\end{gathered}
$$

where $\rho$ the mass density, the comma in subscript indicates partial derivative.

The generalized geometrical equations are

$$
\begin{gathered}
\varepsilon_{k l}=\frac{1}{2}\left(u_{k, l}+u_{l, k}\right) \\
E_{m}=-\varphi_{, m} \\
H_{m}=-\psi, m
\end{gathered}
$$

Combing of Equations (1)-(9), one easily obtains

$$
\begin{aligned}
& \frac{1}{2} C_{i j k l}\left(u_{k, l j}+u_{l, k j}\right)-e_{i j n} \varphi_{, n j}-q_{i j n} \psi_{, n j}=\rho \ddot{u}_{i} \\
& \frac{1}{2} e_{k l n}\left(u_{k, l n}+u_{l, k n}\right)+g_{m n} \varphi_{, m n}+\alpha_{m n} \psi_{, m n}=0 \\
& \frac{1}{2} q_{k l n}\left(u_{k, l n}+u_{l, k n}\right)+\alpha_{m n} \varphi_{, m n}+\mu_{m n} \psi_{, m n}=0
\end{aligned}
$$

Let us consider an anisotropic electric-magneto-elastic plate as shown in Figure $1, x_{1} 0 x_{2}$ is the middle surface of the plate which is infinite in the two directions $x_{1}$ and $x_{2}, x_{3}$ is the thickness direction.

The angle between the incident direction of the wave and the $x_{1}$-axis is assumed to be $\theta$, so that the following relationships can be obtained as

$$
\left.\begin{array}{l}
k_{1}=k \cos \theta \\
k_{2}=k \sin \theta
\end{array}\right\}
$$

where $k$ is wave number in the plane $x_{1} 0 x_{2}$.

The elastic wave tensor can be expressed as the following form 


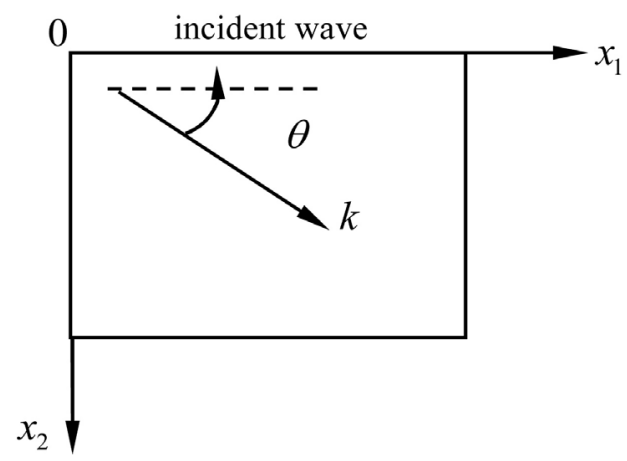

Figure 1. The model of electric-magnetic-elastic plate.

$$
u_{i}=\Phi_{, i}+\varepsilon_{i j k} \Psi_{j, k}
$$

where $\varepsilon_{i j k}$ is a permutation tensor, the scalar field $\Phi$ and the vector field $\Psi_{j}$ are assumed to be

$$
\begin{gathered}
\Phi=f\left(x_{3}\right) \mathrm{e}^{i\left(k_{1} x_{1}+k_{2} x_{2}-\omega t\right)} \\
\Psi_{j}=g_{j}\left(x_{3}\right) \mathrm{e}^{i\left(k_{1} x_{1}+k_{2} x_{2}-\omega t\right)}
\end{gathered}
$$

In Equation (13), $f\left(x_{3}\right)$ is tried to take the following form

$$
f\left(x_{3}\right)=A_{m} \sin \left(\eta_{m}^{\mathrm{d}} x_{3}\right)
$$

where $\eta_{m}^{\mathrm{d}}$ is the $m$ th wave number of the dilatation wave in the thickness direction $x_{3}$.

In Equation (14), $g_{j}\left(x_{3}\right)$ is tried to take the following form

$$
g_{j}\left(x_{3}\right)=F_{j n} \sin \left(\eta_{n}^{\mathrm{s}} x_{3}\right)
$$

where $\eta_{m}^{\mathrm{s}}$ is the $m$ th wave number of the shear wave in the thickness direction.

The electric-magneto-elastic plate is assumed to be polarized in the direction $x_{3}$, the static electric-potential and magneto-potential is tried to take the following forms

$$
\begin{gathered}
\varphi=\left[f_{\varphi}^{\prime}\left(x_{3}\right)+i\left(k_{1} g_{2 \varphi}\left(x_{3}\right)-k_{2} g_{1 \varphi}\left(x_{3}\right)\right)\right] \mathrm{e}^{i\left(k_{1} x_{1}+k_{2} x_{2}-\omega t\right)} \\
\psi=\left[f_{\psi}^{\prime}\left(x_{3}\right)+i\left(k_{1} g_{2 \psi}\left(x_{3}\right)-k_{2} g_{1 \psi}\left(x_{3}\right)\right)\right] \mathrm{e}^{i\left(k_{1} x_{1}+k_{2} x_{2}-\omega t\right)}
\end{gathered}
$$

where

$$
\begin{gathered}
\left\{\begin{array}{l}
f_{\varphi}\left(x_{3}\right)=A_{m}^{\varphi} \sin \left(\eta_{m}^{d} x_{3}\right) \\
f_{\psi}\left(x_{3}\right)=A_{m}^{\psi} \sin \left(\eta_{m}^{d} x_{3}\right)
\end{array}\right. \\
\left\{\begin{array}{l}
g_{j \varphi}\left(x_{3}\right)=F_{j n}^{\varphi} \sin \left(\eta_{n}^{\mathrm{s}} x_{3}\right) \\
g_{j \psi}\left(x_{3}\right)=F_{j n}^{\psi} \sin \left(\eta_{n}^{\mathrm{s}} x_{3}\right)
\end{array}\right.
\end{gathered}
$$

\section{Numeric Example and Discussion}

In the subsequent numeric example, the electric-magnetic-elastic plate is as- 
sumed to be polarized in the direction $x_{3}$, the thickness of the plate is $2 h$, the mass density $\rho=7454 \mathrm{~kg} / \mathrm{m}^{3}$, the material parameters are given by Tables 1-3.

Except these parameters given by Tables 1-3, the others are assumed to be zeros.

\subsection{Mixed Boundary Condition}

For smooth rigid boundary conditions, namely, $\left.u_{3}\right|_{x_{3}= \pm h}=0$, $\left.\sigma_{23}\right|_{x_{3}= \pm h}=\left.\sigma_{31}\right|_{x_{3}= \pm h}=0,\left.\varphi\right|_{x_{3}= \pm h}=0,\left.D_{1}\right|_{x_{3}= \pm h}=\left.D_{2}\right|_{x_{3}= \pm h}=0,\left.\psi\right|_{x_{3}= \pm h}=0$,

$\left.B_{1}\right|_{x_{3}= \pm h}=\left.B_{2}\right|_{x_{3}= \pm h}=0, \eta_{m}^{\mathrm{d}}$ and $\eta_{m}^{\mathrm{s}}$ which satisfy the boundary condition are taken as the following forms

$$
\begin{gathered}
\eta_{m}^{\mathrm{d}}=\frac{m \pi}{2 h}, \quad m=1,3,5, \cdots \\
\eta_{n}^{\mathrm{s}}=\frac{n \pi}{2 h}, \quad n=0,2,4,6, \cdots
\end{gathered}
$$

\subsection{Dynamic Equilibrium Equations}

For the numeric example, Equations (10a)-(10c) can be rewritten as

$$
\begin{aligned}
& C_{1111} u_{1,11}+C_{1122} u_{2,21}+C_{1133} u_{3,31}+\frac{1}{2} C_{1212}\left(u_{2,12}+u_{1,22}\right) \\
& +\frac{1}{2} C_{3131}\left(u_{3,13}+u_{1,33}\right)-e_{111} \varphi_{, 11}-e_{311} \varphi_{, 31}-q_{311} \psi_{, 31}-q_{111} \psi_{, 11}=\rho \ddot{u}_{1} \\
& C_{2211} u_{1,12}+C_{2222} u_{2,22}+C_{2233} u_{3,32}+\frac{1}{2} C_{2121}\left(u_{2,11}+u_{1,21}\right) \\
& +\frac{1}{2} C_{2323}\left(u_{2,33}+u_{3,23}\right)-e_{222} \varphi_{, 22}-e_{223} \varphi_{, 23}-q_{222} \psi_{, 22}-q_{223} \psi_{, 23}=\rho \ddot{u}_{2}
\end{aligned}
$$

Table 1. Elastic parameters, unit (GPa).

\begin{tabular}{ccccccc}
\hline$C_{1111}=C_{2222}$ & $C_{1122}=C_{1133}$ & $C_{2211}=C_{2233}$ & $C_{3311}=C_{3322}$ & $C_{3333}$ & $C_{1212}=C_{2121}$ & $C_{2323}=C_{3131}$ \\
\hline 79.7 & 35.8 & 35.8 & 35.8 & 66.8 & 17.2 & 14.4
\end{tabular}

Table 2. Piezoelectric parameters, unit $\left(\mathrm{C} / \mathrm{m}^{2}\right)$; Piezomagnetic parameters, unit $\left(\mathrm{Vs} / \mathrm{m}^{2}\right)$.

\begin{tabular}{cccccc}
\hline \multicolumn{2}{c}{ Piezoelectric parameters, unit $\left(\mathrm{C} / \mathrm{m}^{2}\right)$} & \multicolumn{3}{c}{ Piezomagnetic parameters, unit $\left(\mathrm{Vs} / \mathrm{m}^{2}\right)$} \\
\hline$e_{111}=e_{222}$ & $e_{333}$ & $e_{232}=e_{311}$ & $q_{111}=q_{222}$ & $q_{333}$ & $q_{232}=q_{311}$ \\
\hline-5.9 & 15.2 & 10.5 & -60.9 & 156.8 & 108.3 \\
\hline
\end{tabular}

Table 3. Dielectric parameters, unit (As/Am); Magnetic permeability parameters, unit (Vs/Am)

\begin{tabular}{cccccc}
\hline \multicolumn{2}{c}{ Dielectric parameters, unit (As/Am) } & \multicolumn{3}{c}{ Magnetic permeability parameters, unit (Vs/Am) } \\
\hline$g_{111}$ & $g_{222}$ & $g_{333}$ & $\mu_{111}$ & $\mu_{222}$ & $\mu_{333}$ \\
\hline $3.8 \times 10^{-10}$ & $3.8 \times 10^{-10}$ & $3.8 \times 10^{-10}$ & $5.4 \times 10^{-6}$ & $5.4 \times 10^{-6}$ & $5.4 \times 10^{-6}$ \\
\hline
\end{tabular}




$$
\begin{aligned}
C_{3311} u_{1,13} & +C_{3322} u_{2,23}+C_{3333} u_{3,33}+\frac{1}{2} C_{3131}\left(u_{3,11}+u_{1,31}\right)+\frac{1}{2} C_{3232}\left(u_{3,22}+u_{2,32}\right) \\
-e_{333} \varphi_{, 33} & -e_{232} \varphi_{, 23}-e_{311} \varphi_{, 13}-q_{333} \psi_{, 33}-q_{232} \psi_{, 23}-q_{311} \psi_{, 13}=\rho \ddot{u}_{3} \\
& \frac{1}{2} e_{311}\left(u_{3,11}+u_{1,31}\right)+\frac{1}{2} e_{232}\left(u_{2,32}+u_{3,22}\right)+e_{111} u_{1,13}+e_{222} u_{2,23} \\
& +e_{333} u_{3,33}+g_{111} \varphi_{, 11}+g_{222} \varphi_{, 22}+g_{333} \varphi_{, 33}=0 \\
& \frac{1}{2} q_{311}\left(u_{3,11}+u_{1,31}\right)+\frac{1}{2} q_{232}\left(u_{2,32}+u_{3,22}\right)+q_{111} u_{1,13}+q_{222} u_{2,23} \\
+ & q_{333} u_{3,33}+\mu_{111} \psi_{, 11}+\mu_{222} \psi_{, 22}+\mu_{333} \psi_{, 33}=0
\end{aligned}
$$

Substituting of Equations (12), (17) and (18) into Equations (23)-(27), gives

$$
\begin{aligned}
& -C_{1111} k_{1}^{2} u_{1}-C_{1122} k_{1} k_{2} u_{2}+C_{1133} i k_{1} u_{3,3}-\frac{1}{2} C_{1212}\left(k_{1}^{2} u_{2}+k_{1} k_{2} u_{1}\right) \\
& -\frac{1}{2} C_{3131}\left(k_{1}^{2} u_{3}-i k_{1} u_{1,3}\right)-e_{111} i k_{1} \varphi_{, 3}+e_{311} k_{1}^{2} \varphi+q_{311} k_{1}^{2} \psi \\
& -q_{111} i k_{1} \psi_{, 3}=-\rho \omega^{2} u_{1} \\
& -C_{2211} k_{1} k_{2} u_{1}-C_{2222} k_{2}^{2} u_{2}+C_{2233} i k_{2} u_{3,3}-\frac{1}{2} C_{2121}\left(k_{1} k_{2} u_{2}+k_{2}^{2} u_{1}\right) \\
& -\frac{1}{2} C_{2323}\left(-i k_{2} u_{2,3}+k_{2}^{2} u_{3}\right)-e_{222} i k_{2} \varphi_{, 3}+e_{232} k_{2}^{2} \varphi+q_{232} k_{2}^{2} \psi \\
& -q_{222} i k_{2} \psi_{, 3}=-\omega^{2} \rho u_{2} \\
& C_{3311} i k_{1} u_{1,3}+C_{3322} i k_{2} u_{2,3}+C_{3333} u_{3,33}+\frac{1}{2} C_{3131}\left(i k_{1} u_{3,3}+u_{1,33}\right) \\
& +\frac{1}{2} C_{2323}\left(i k_{2} u_{3,3}+u_{2,33}\right)-e_{333} \varphi_{, 33}-e_{232} i k_{2} \varphi_{, 3}-e_{311} i k_{1} \varphi_{, 3} \\
& -q_{333} \psi_{, 33}-q_{232} i k_{2} \psi_{, 3}-q_{311} i k_{1} \psi_{, 3}=-\rho \omega^{2} u_{3} \\
& -\frac{1}{2} e_{311}\left(k_{1}^{2} u_{3}-i k_{1} u_{1,3}\right)-\frac{1}{2} e_{232}\left(-i k_{2} u_{2,3}+k_{2}^{2} u_{3}\right)+e_{111} i k_{1} u_{1,3} \\
& +e_{222} i k_{2} u_{2,3}+e_{333} u_{3,33}-g_{111} k_{1}^{2} \varphi-g_{222} k_{2}^{2} \varphi+g_{333} \varphi_{, 33}=0 \\
& -\frac{1}{2} q_{311}\left(k_{1}^{2} u_{3}-i k_{1} u_{1,3}\right)-\frac{1}{2} q_{232}\left(-i k_{2} u_{2,3}+k_{2}^{2} u_{3}\right)+q_{111} i k_{1} u_{1,3} \\
& +q_{222} i k_{2} u_{2,3}+q_{333} u_{3,33}-\mu_{111} k_{1}^{2} \psi-\mu_{222} k_{2}^{2} \psi+\mu_{333} \psi, 33=0
\end{aligned}
$$

The following equations can be obtained by using the orthogonality of the trigonometric function for Equations (28)-(32) and by dealing with the real and imaginary parts of Equations (28)-(32).

$$
\begin{gathered}
C_{3131} A_{m} \cos \frac{m \pi x_{3}}{2 h}=e_{311} A_{m}^{\varphi} \cos \frac{m \pi x_{3}}{2 h}+q_{311} A_{m}^{\psi} \cos \frac{m \pi x_{3}}{2 h} \\
C_{2323} A_{m} \cos \frac{m \pi x_{3}}{2 h}=e_{232} A_{m}^{\varphi} \cos \frac{m \pi x_{3}}{2 h}+q_{232} A_{m}^{\psi} \cos \frac{m \pi x_{3}}{2 h} \\
\left(C_{3131} k_{1}+C_{2323} k_{2}\right) A_{m} \cos \frac{m \pi x_{3}}{2 h}=e_{311} k_{1} A_{m}^{\varphi} \cos \frac{m \pi x_{3}}{2 h}+q_{311} k_{1} A_{m}^{\psi} \cos \frac{m \pi x_{3}}{2 h} \\
{\left[\left(e_{311}+e_{111}\right) k_{1}^{2}+\left(e_{232}+e_{222}\right) k_{2}^{2}+e_{333}\left(\eta_{m}^{\mathrm{d}}\right)^{2}\right] A_{m} \cos \frac{m \pi x_{3}}{2 h}} \\
=-\left[g_{111} k_{1}^{2}+g_{222} k_{2}^{2}+g_{333}\left(\eta_{m}^{\mathrm{d}}\right)^{2}\right] A_{m}^{\varphi} \cos \frac{m \pi x_{3}}{2 h}
\end{gathered}
$$




$$
\begin{aligned}
& {\left[\left(q_{311}+q_{111}\right) k_{1}^{2}+\left(q_{232}+q_{222}\right) k_{2}^{2}+e_{333}\left(\eta_{m}^{\mathrm{d}}\right)^{2}\right] A_{m} \cos \frac{m \pi x_{3}}{2 h}} \\
& =-\left[\mu_{111} k_{1}^{2}+\mu_{222} k_{2}^{2}+\mu_{333}\left(\eta_{m}^{\mathrm{d}}\right)^{2}\right] A_{m}^{\psi} \cos \frac{m \pi x_{3}}{2 h} \\
& \left(-C_{1111} k_{1}^{2}-C_{1122} k_{2}^{2}-C_{1133}\left(\eta_{m}^{\mathrm{d}}\right)^{2}-C_{1212} k_{1} k_{2}\right) A_{m}+e_{111}\left(\eta_{m}^{\mathrm{d}}\right)^{2} A_{m}^{\varphi} \\
& +q_{111}\left(\eta_{m}^{\mathrm{d}}\right)^{2} A_{m}^{\psi}=-\rho \omega^{2} A_{m} \\
& \left(-C_{2211} k_{1}^{2}-C_{2222} k_{2}^{2}-C_{2233}\left(\eta_{m}^{\mathrm{d}}\right)^{2}-C_{2121} k_{1} k_{2}\right) A_{m}+e_{222}\left(\eta_{m}^{\mathrm{d}}\right)^{2} A_{m}^{\varphi} \\
& +q_{222}\left(\eta_{m}^{\mathrm{d}}\right)^{2} A_{m}^{\psi}=-\rho \omega^{2} A_{m} \\
& \left(-C_{3311} k_{1}^{2}-C_{3322} k_{2}^{2}-C_{3333}\left(\eta_{m}^{\mathrm{d}}\right)^{2}\right) A_{m}+e_{333}\left(\eta_{m}^{\mathrm{d}}\right)^{2} A_{m}^{\varphi} \\
& +q_{333}\left(\eta_{m}^{\mathrm{d}}\right)^{2} A_{m}^{\psi}=-\rho \omega^{2} A_{m} \\
& a_{11} F_{1 n}+a_{12} F_{2 n}+a_{13} F_{3 n}+a_{14} F_{1 n}^{\varphi}+a_{15} F_{2 n}^{\varphi}+a_{16} F_{1 n}^{\psi}+a_{17} F_{2 n}^{\psi}=0 \\
& a_{21} F_{1 n}+a_{22} F_{2 n}+a_{23} F_{3 n}+a_{24} F_{1 n}^{\varphi}+a_{25} F_{2 n}^{\varphi}+a_{26} F_{1 n}^{\psi}+a_{27} F_{2 n}^{\psi}=0 \\
& a_{31} F_{1 n}+a_{32} F_{2 n}+a_{33} F_{3 n}+a_{34} F_{1 n}^{\varphi}+a_{35} F_{2 n}^{\varphi}+a_{36} F_{1 n}^{\psi}+a_{37} F_{2 n}^{\psi}=0 \\
& a_{41} F_{1 n}+a_{42} F_{2 n}+a_{43} F_{3 n}+a_{44} F_{1 n}^{\varphi}+a_{45} F_{2 n}^{\varphi}+a_{46} F_{1 n}^{\psi}+a_{47} F_{2 n}^{\psi}=0 \\
& a_{51} F_{1 n}+a_{52} F_{2 n}+a_{53} F_{3 n}+a_{54} F_{1 n}^{\varphi}+a_{55} F_{2 n}^{\varphi}+a_{56} F_{1 n}^{\psi}+a_{57} F_{2 n}^{\psi}=0 \\
& a_{61} F_{1 n}+a_{62} F_{2 n}+a_{63} F_{3 n}+a_{64} F_{1 n}^{\varphi}+a_{65} F_{2 n}^{\varphi}+a_{66} F_{1 n}^{\psi}+a_{67} F_{2 n}^{\psi}=0 \\
& a_{71} F_{1 n}+a_{72} F_{2 n}+a_{73} F_{3 n}+a_{74} F_{1 n}^{\varphi}+a_{75} F_{2 n}^{\varphi}+a_{76} F_{1 n}^{\psi}+a_{77} F_{2 n}^{\psi}=0 \\
& a_{81} F_{1 n}\left(\eta_{n}^{\mathrm{s}}\right) \cos \left(\eta_{n}^{\mathrm{s}} x_{3}\right)+a_{82} F_{2 n}\left(\eta_{n}^{\mathrm{s}}\right) \cos \left(\eta_{n}^{\mathrm{s}} x_{3}\right)+a_{83} F_{3 n}\left(\eta_{n}^{\mathrm{s}}\right) \cos \left(\eta_{n}^{\mathrm{s}} x_{3}\right) \\
& +a_{84} F_{1 m}^{\varphi}\left(\eta_{m}^{\mathrm{s}}\right) \cos \left(\eta_{m}^{\mathrm{s}} x_{3}\right)+a_{85} F_{2 m}^{\varphi}\left(\eta_{m}^{\mathrm{s}}\right) \cos \left(\eta_{n}^{\mathrm{s}} x_{3}\right) \\
& +a_{86} F_{1 n}^{\psi}\left(\eta_{n}^{\mathrm{s}}\right) \cos \left(\eta_{n}^{\mathrm{s}} X_{3}\right)+a_{87} F_{2 n}^{\psi}\left(\eta_{n}^{\mathrm{s}}\right) \cos \left(\eta_{n}^{\mathrm{s}} x_{3}\right)=0
\end{aligned}
$$

The above Equations (32)-(40) do not contain the shear term $\eta_{n}^{\mathrm{s}}$, so they are P-wave equations. Equations (41)-(48) are not related to the dilatation wave term $\eta_{m}^{\mathrm{d}}$, so they are S-wave equations. This indicates that the kind of wave is invariable after reflection. Equations (33)-(37) and Equations (48) are true according to Saint-Venant's principle. $a_{i j}$ in Equations (41)-(48) are given by Appendix A.

\section{Wave Characteristics}

Equations (38)-(40) about the longitudinal wave can be written in the following matrix form

$$
\left[\begin{array}{ccc}
b_{11}-\rho \omega^{2} & -b_{12} & -b_{13} \\
b_{21}-\rho \omega^{2} & -b_{22} & -b_{23} \\
b_{31}-\rho \omega^{2} & -b_{32} & -b_{33}
\end{array}\right]\left\{\begin{array}{c}
A_{m} \\
A_{m}^{\varphi} \\
A_{m}^{\psi}
\end{array}\right\}=\left\{\begin{array}{l}
0 \\
0 \\
0
\end{array}\right\}
$$

For Equation (49) to have a nontrivial solution, it is required that 


$$
\left|\begin{array}{lll}
b_{11}-\rho \omega^{2} & -b_{12} & -b_{13} \\
b_{21}-\rho \omega^{2} & -b_{22} & -b_{23} \\
b_{31}-\rho \omega^{2} & -b_{32} & -b_{33}
\end{array}\right|=0
$$

where $b_{i j}(i, j=1,2,3)$ are given by Appendix B.

The relationship of wave number $p=\sqrt{k^{2}+m^{2}}$ and circle frequencies $\omega$ can be obtained by solving Equation (50), and then phase velocity is defined as

$$
c=\frac{\omega}{p}
$$

and phase velocity surface

$$
f=c(\theta, \omega, p)=0
$$

can be obtained.

Before plotting the figures, we introduce the dimensionless frequency $\bar{\omega}=\omega / c_{p}$, phase wave velocity $\bar{c}=c / c_{p}$, where $c_{p}=\sqrt{c_{1111} / \rho}$.

Figure 2 shows the dispersion spectrum of the electric-magneto-elastic plate (EMEP) about longitudinal waves with $m=1,7,13$ and 19. The dispersion curves affected by the geometric boundaries and material properties are compared in detail in Figures 2(a)-(c). The four Figures are, respectively, the phase

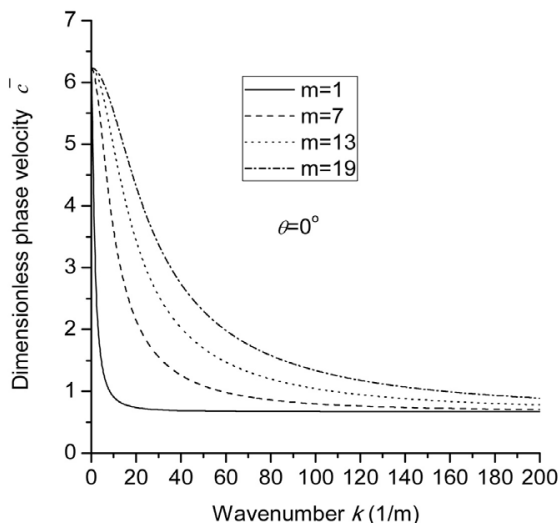

(a)

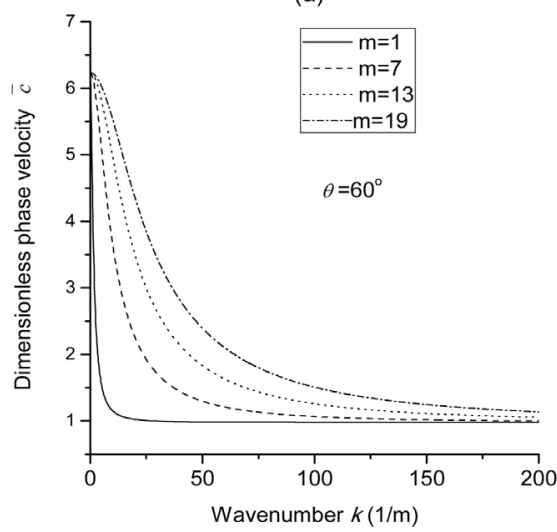

(c)

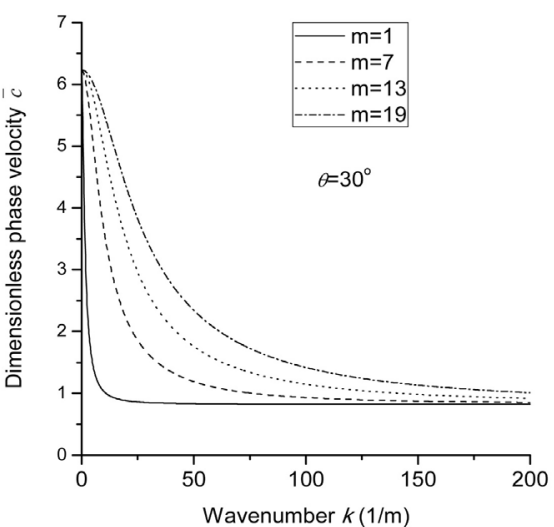

(b)

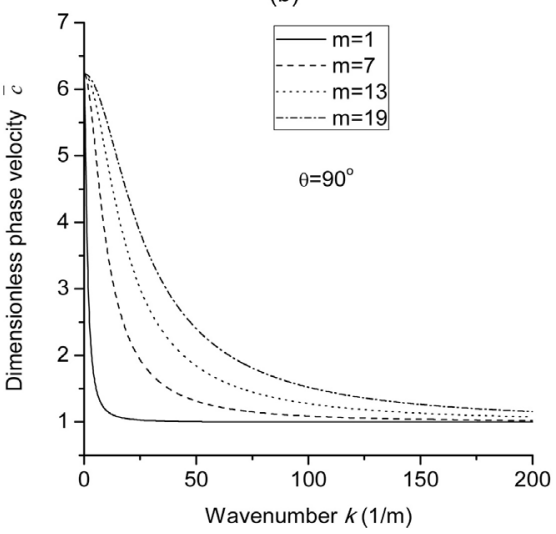

(d)

Figure 2. Dimensionless phase velocity curves of dilatation wave in the electric-magnetoelastic plate when the incident angle $\theta$ is $0^{\circ}$ (a), $\theta$ is $30^{\circ}$ (b), $\theta$ is $60^{\circ}$ (c) and $\theta$ is $90^{\circ}$ (d). 
velocity curves in the different incident direction $\theta$. It can be found from the four Figures that with the increase of $m$, the dispersion of wave becomes more and more serious. The larger $m$ is, the closer the direction of wave propagation is to the thickness of the plate, this means that the closer the wave propagation direction is to the direction $x_{3}$, the more dispersive it is. This can be explained as that the geometrical and material dispersion of longitudinal wave propagating along the thickness direction is more serious than propagating in middle plane.

Figure 3 shows the phase velocity surfaces of EMEP about longitudinal waves with the wave number $m=3$ and $k=2,20,200$. These left graphs and the right ones are drawn in the absolute and relative coordinate systems, respectively. It

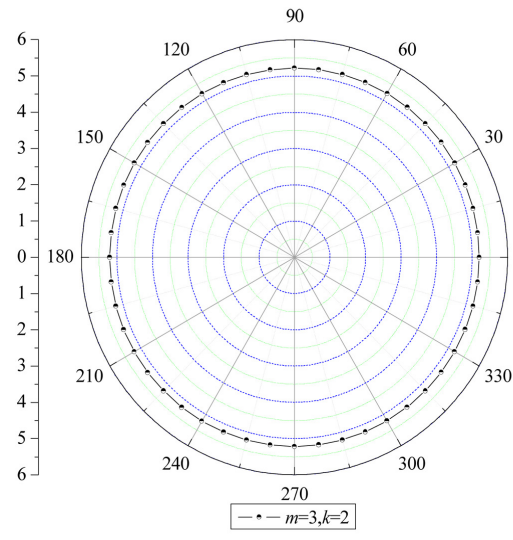

(a)

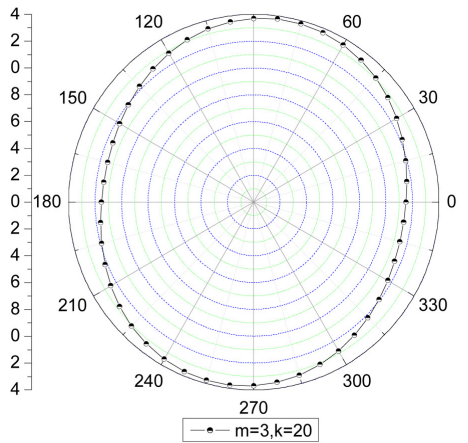

(c)

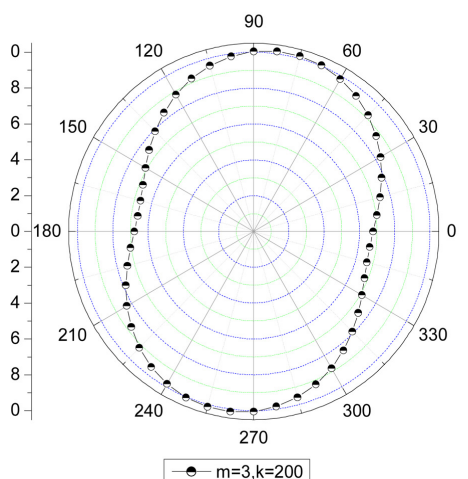

(e)

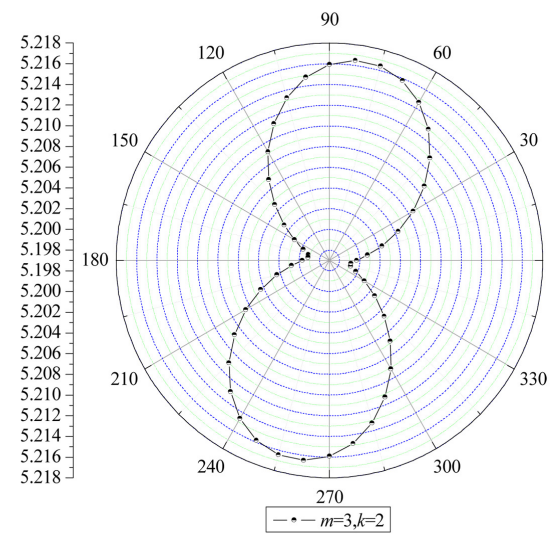

(b)

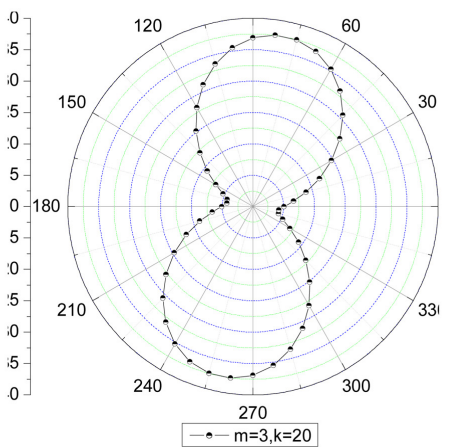

(d)

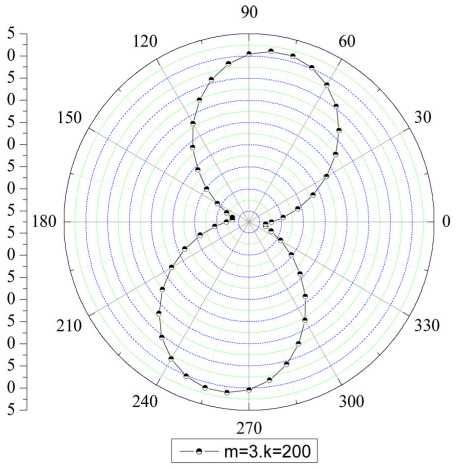

(f)

Figure 3. Phase velocity surface of longitudinal wave in the electric-magneto-elastic plate when $m=3, k=2,20,200$. 
can be seen from comparison of the left Figures of Figures 3(a)-(c) that the phase velocity surface changes from an approximate circular to an approximate ellipse as the wave number $k$ changes from 2 to 200. This is due to the anisotropy in the $x_{1} 0 x_{2}$ plane caused by the polarization of the electromagnetic field along the $X_{3}$-axis. It can be found from right Figures of Figure 3 that the phase velocity surfaces are symmetrical about $\theta=75^{\circ}$ and $\theta=165^{\circ}$, this means that EMEP is approximately orthotropic.

To further clarify the conclusions obtained from Figure 3. Without loss of generality, Figure 4 is drawn when the representative wave numbers $m=51$ and $k=20,200,2000$ are taken. The same conclusions can be obtained from Figure 4 as these from Figure 3.

The matrix form of Equations (41)-(47) about the shear wave can be written as

$$
\left[\begin{array}{lllllll}
a_{11} & a_{12} & a_{13} & a_{14} & a_{15} & a_{16} & a_{17} \\
a_{21} & a_{22} & a_{23} & a_{24} & a_{25} & a_{26} & a_{27} \\
a_{31} & a_{32} & a_{33} & a_{34} & a_{35} & a_{36} & a_{37} \\
a_{41} & a_{42} & a_{43} & a_{44} & a_{45} & a_{46} & a_{47} \\
a_{51} & a_{52} & a_{53} & a_{54} & a_{55} & a_{56} & a_{57} \\
a_{61} & a_{62} & a_{63} & a_{64} & a_{65} & a_{66} & a_{67} \\
a_{71} & a_{72} & a_{73} & a_{74} & a_{75} & a_{76} & a_{77}
\end{array}\right]\left\{\begin{array}{l}
F_{1 n} \\
F_{2 n} \\
F_{3 n} \\
F_{1 n}^{\varphi} \\
F_{2 n}^{\varphi} \\
F_{1 n}^{\psi} \\
F_{2 n}^{\psi}
\end{array}\right\}=\left\{\begin{array}{l}
0 \\
0 \\
0 \\
0 \\
0 \\
0 \\
0
\end{array}\right\}
$$

The condition of the nontrivial solution of Equation (53) is that the coefficient determinant of Equation (53) must be zero.

$$
\left|\begin{array}{lllllll}
a_{11} & a_{12} & a_{13} & a_{14} & a_{15} & a_{15} & a_{17} \\
a_{21} & a_{22} & a_{23} & a_{24} & a_{25} & a_{26} & a_{27} \\
a_{31} & a_{32} & a_{33} & a_{34} & a_{35} & a_{36} & a_{37} \\
a_{41} & a_{42} & a_{43} & a_{44} & a_{45} & a_{46} & a_{47} \\
a_{51} & a_{52} & a_{53} & a_{54} & a_{55} & a_{56} & a_{57} \\
a_{61} & a_{62} & a_{63} & a_{64} & a_{65} & a_{66} & a_{67} \\
a_{71} & a_{72} & a_{73} & a_{74} & a_{75} & a_{76} & a_{77}
\end{array}\right|=0
$$

Before plotting the Figures, we introduce the dimensionless frequency $\bar{\omega}=\omega / c_{s}$, the phase wave velocity $\bar{c}=c / c_{s}$ and the group velocity $C_{g}=c_{g} / c_{s}$, where $c_{s}=\sqrt{c_{1313} / \rho}$. The symbols of EMEP and EP denote the electric-magnetic-elastic plate and the elastic plate, respectively.

In the subsequent example, let $n=2, \theta=0^{\circ}, 30^{\circ}, 60^{\circ}$ and $90^{\circ}$ in Equations (54) and (56). The dimensionless circle frequency and the phase velocity corresponding to the different wave numbers $k$ can be obtained from the solution of Equation (54) and Equation (56), respectively. For the purpose of investigation to the effects of the piezoelectric and the piezomagnetic parameters on the dispersion characteristics of the shear waves, we consider the EMEP and the elastic plate EP with the same modulus of elasticity as the former. The dispersion curves of shear waves with the different incident angles $\theta$, namely, the relationship curves of the dimensionless circle frequency $\omega$ and the wave number $k$, are 


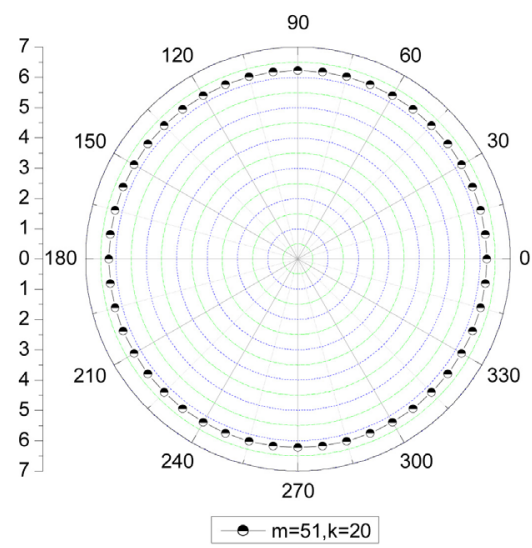

(a)

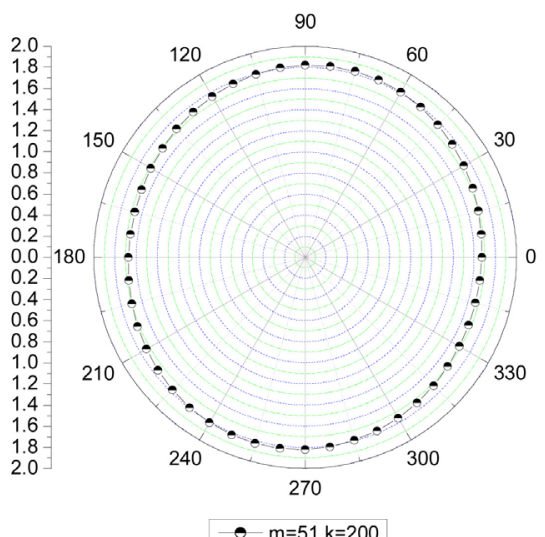

(c)

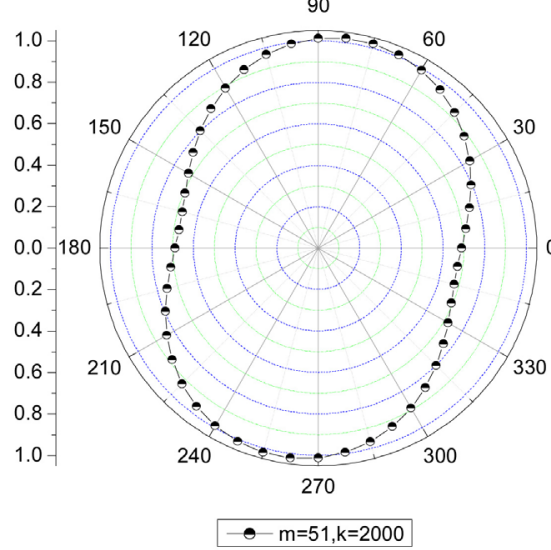

(e)

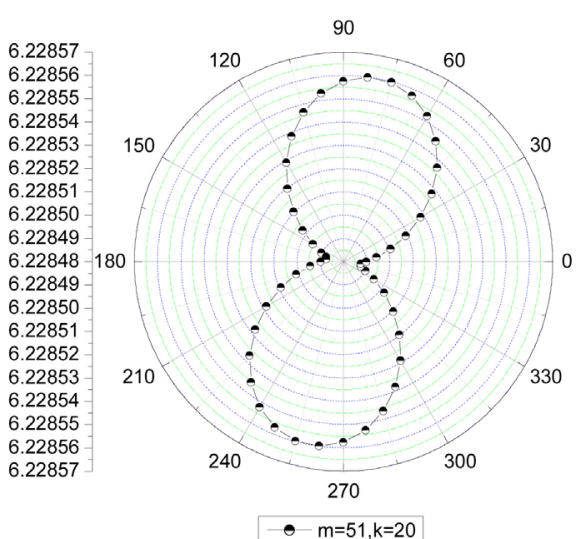

(b)

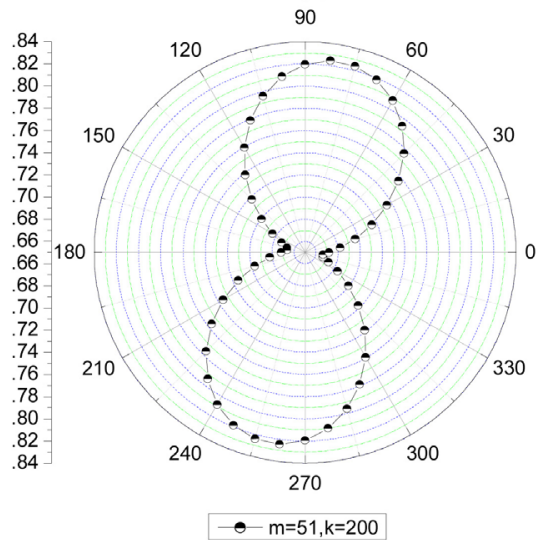

(d)

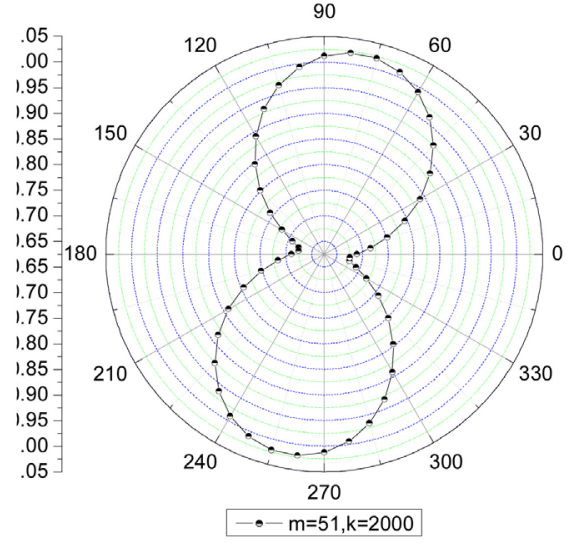

(f)

Figure 4. Phase velocity surface of longitudinal wave in the electric-magneto-elastic plate when $m=51, k=20,200$ and 2000 .

plotted in Figures 5(a)-8(a). The relationship curves of the dimensionless phase velocity $C$ and the wave number $k$ are plotted in Figures 5(b)-8(b).

When the incident angle $\theta$ is $0^{\circ}$, the relationship curves between the dimensionless circle frequencies and the wave numbers for EP and EMEP are shown in Figure 5(a). The phase velocity dispersion curves corresponding to them are shown in Figure 5(b). It can be seen from Figure 5(a) and Figure 5(b) that the shear waves in EP and EMEP are dispersive. As can be seen, the dispersion 


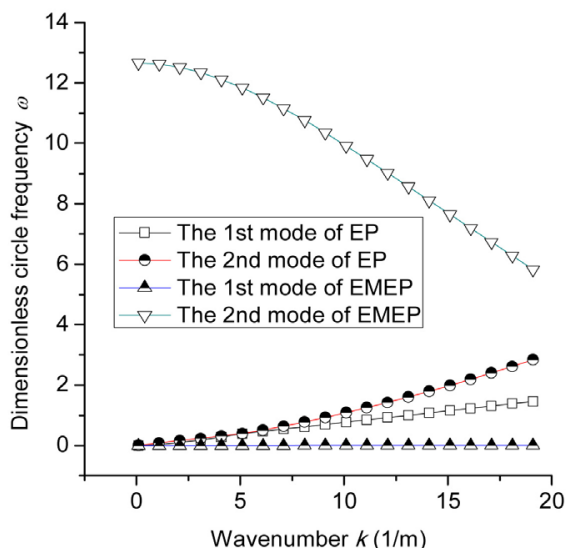

(a)

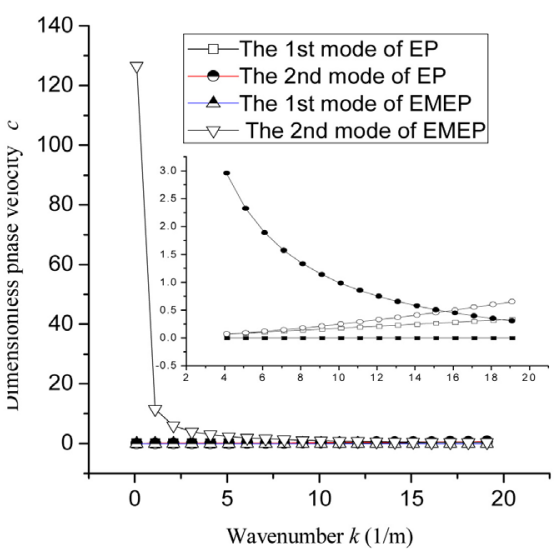

(b)

Figure 5. Dispersion curves of the different wave modes for EP and EME when $\theta$ is $0^{\circ}$.

curves of waves in the two plates are different, especially that of the second wave mode. This indicates that the dispersion characteristics of wave are affected by the piezoelectric and piezomagnetic parameters. It also can be found from Figure 5(b) that effect of the piezoelectric and the piezomagnetic parameters on the phase velocities of the shear waves with the small wave number $k$ is very obvious. The smaller $k$ is, the closer the propagation direction of shear wave is to the thickness direction of the plate. This means that the dispersion of shear wave propagating along the thickness direction of the plate is more serious than that along the middle plane of the plate.

When $\theta$ is $30^{\circ}$, the dispersion curves of the different wave modes for EP and EMEP are shown in Figure 6. It can be found from the comparison of Figure 6(a) with Figure 5(a) that the dispersion characteristics of the waves propagating along the direction $\theta=30^{\circ}$ is different from that along the direction $\theta=0^{\circ}$, it is cause that EMEP is anisotropic. It can also be seen from Figure 6(a) that the piezoelectric and piezomagnetic parameters have a great influence on the dispersion characteristics of the various shear wave modes, and from Figure 6(b) that the piezoelectric and piezomagnetic parameters have a great influence on the phase velocity of shear waves with the small wave number $k$, especially that of the third wave mode.

When the incident angle $\theta$ is $60^{\circ}$, the dispersion curves of the different wave modes for EP and EMEP are shown in Figure 7. It can be found from the comparisons of Figure 7(a) with both Figure 5(a) and Figure 6(a) that the dispersion curves in Figure 7(a) are different from these in Figure 5(a) and Figure 6(a), it can be also seen from Figure 7(a) that piezoelectric and piezomagnetic parameters have a great influence on the dispersion characteristics of various shear wave modes, from Figure 7(b) that the piezoelectric and piezomagnetic parameters have a great influence on the phase velocity of the shear waves with the small wave number $k$, especially that of the third wave mode.

When the incident angle $\theta$ is $90^{\circ}$, the dispersion curves of the different shear wave modes for EP and EMEP are shown in Figure 8. The shear wave propagating along the incident angle $90^{\circ}$ is in reality $2-\mathrm{D}$ wave. It can be found from 


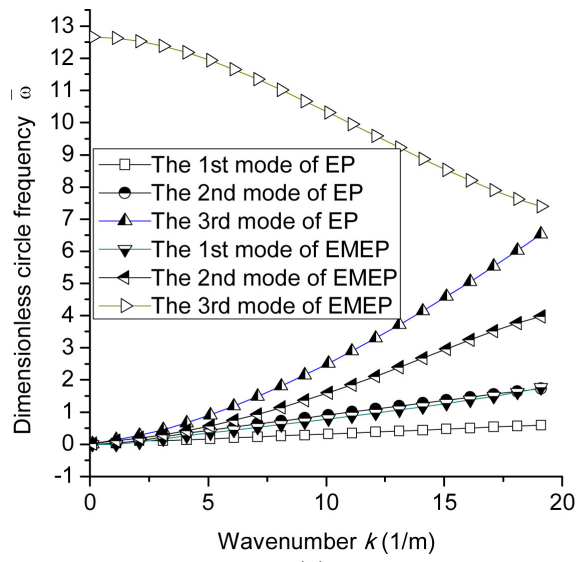

(a)

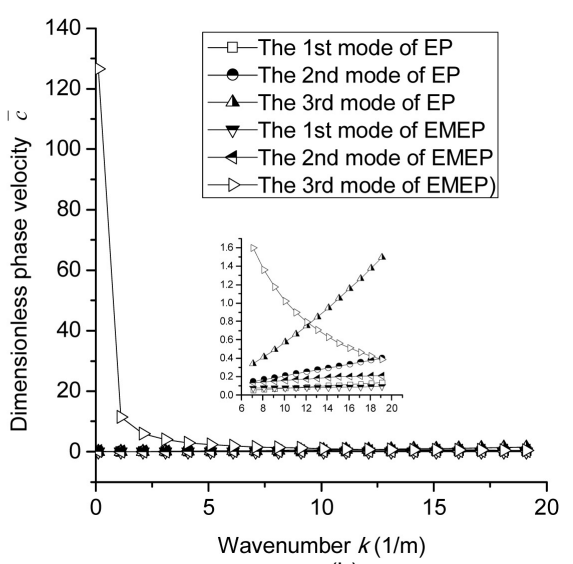

(b)

Figure 6. Dispersion curves of the different wave modes for the EP and the EMEP when $\theta$ is $30^{\circ}$.

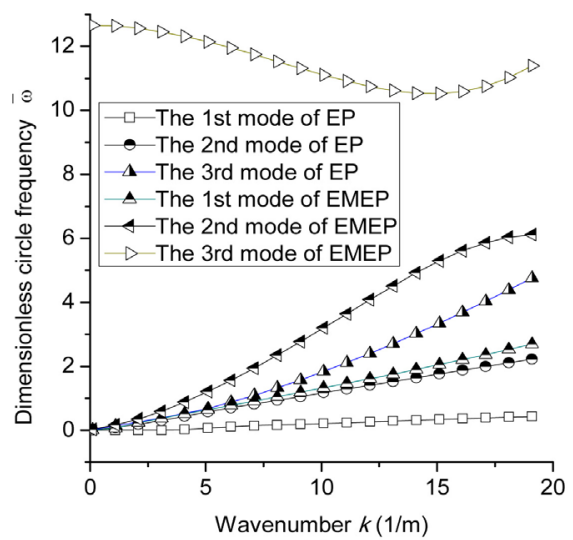

(a)

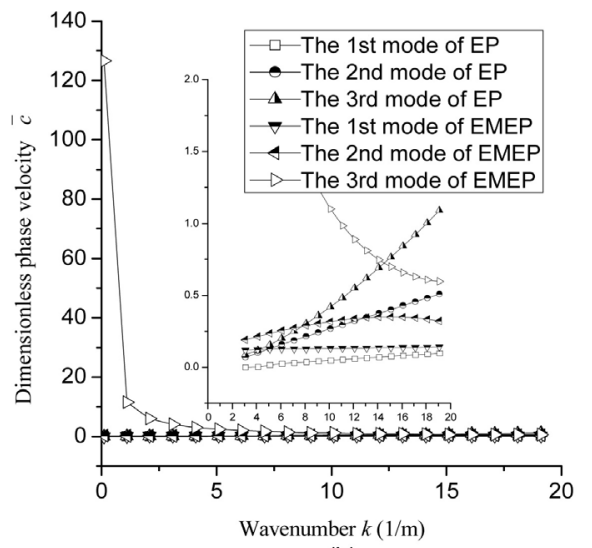

(b)

Figure 7. Dispersion curves of the different wave modes for EP and EMEP when $\theta$ is $60^{\circ}$.

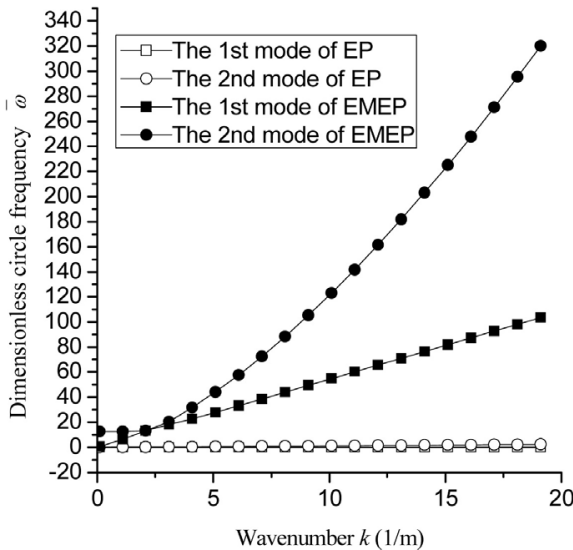

(a)

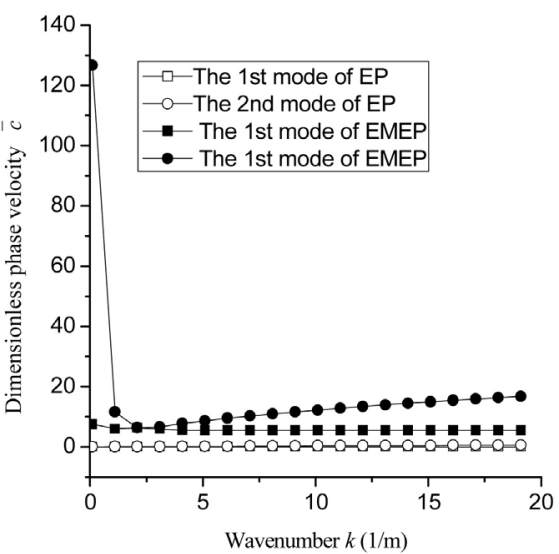

(b)

Figure 8. Dispersion curves of the different wave modes for EP and EMEP when $\theta$ is $90^{\circ}$.

Figure 8 (a) that the piezoelectric and piezomagnetic parameters have a great influence on the dispersion characteristics of the various shear wave modes, and from Figure 8 (b) that the piezoelectric and piezomagnetic parameters have a 
great influence on the phase velocity of the shear waves with the small wave number $k$, especially that of the second wave mode.

The phase velocity surface of the third shear wave mode for EMEP is shown in Figure 9 when the wave number $n=2$ and $k=2,20$ and 40. Figure 9(a), Figure 9(c), Figure 9(e) are drawn in the absolute coordinate, Figure 9(b), Figure 9(d), Figure 9(f) are drawn in the relative coordinate. By comparing Figure 9(a), Figure 9(c), Figure 9(e), it can be seen that the phase velocity surface changes from an approximate circle to a non-circle as the wave number $k$

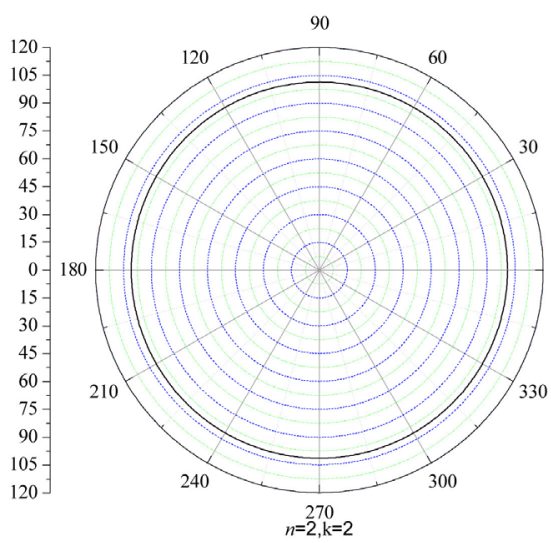

(a)
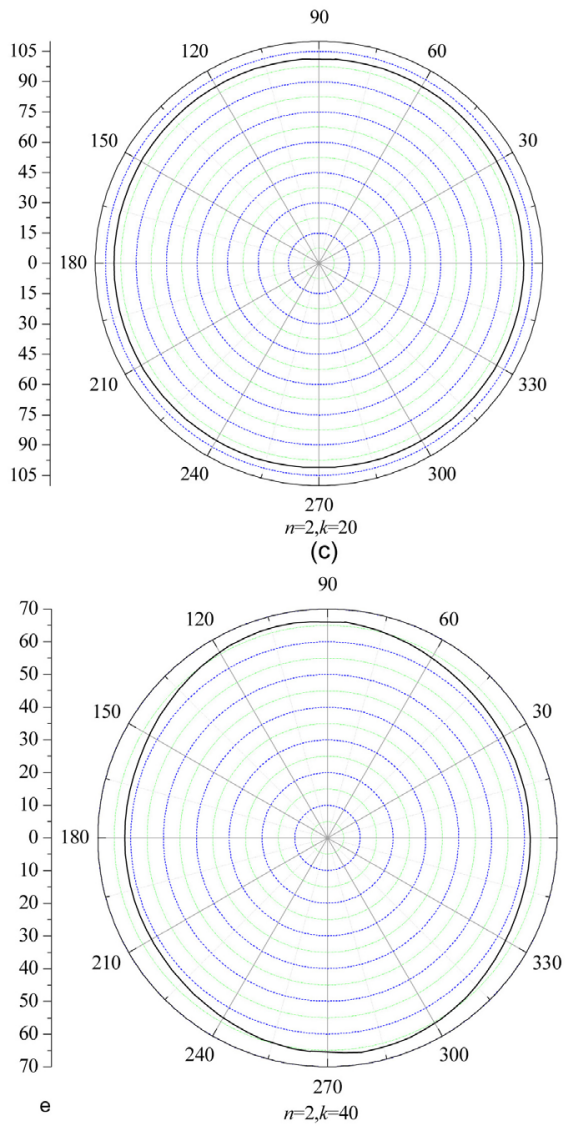

(e)
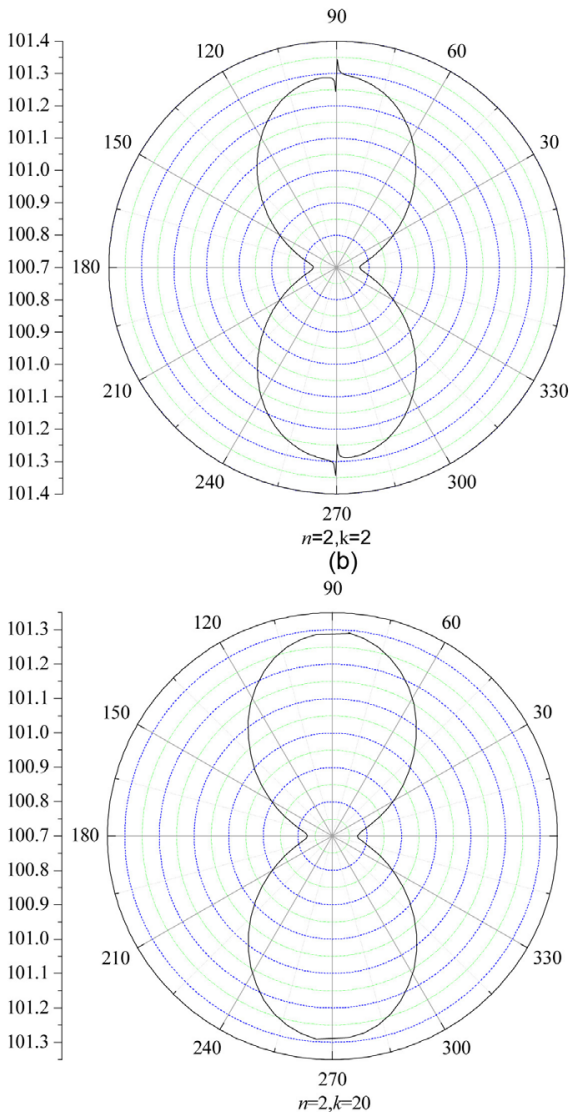

(d)

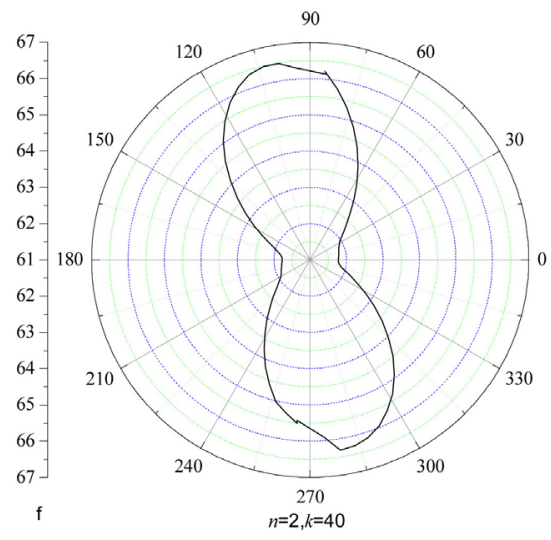

(f)

Figure 9. Phase velocity surface of shear wave in EMEP when $n=2, k=2,20$ and 40. 


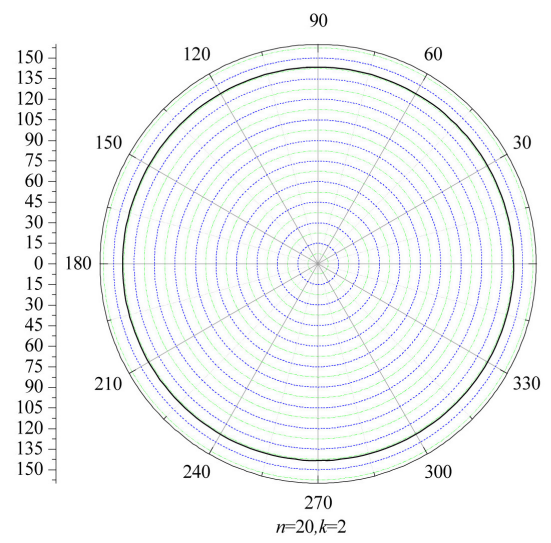

(a)

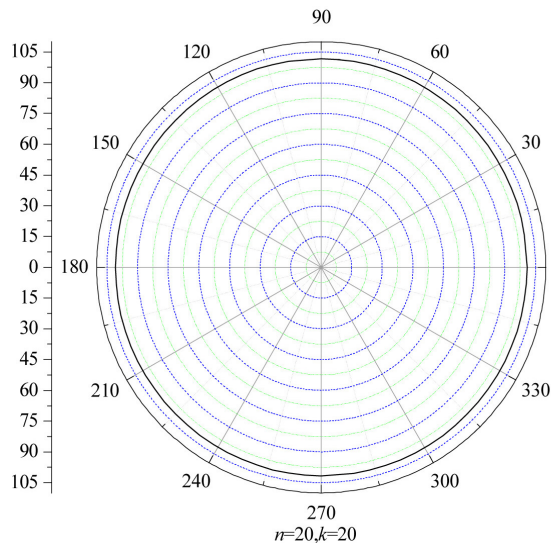

(c)

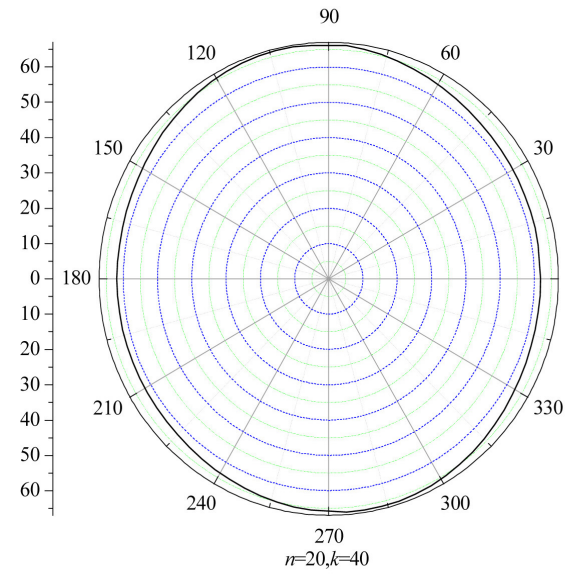

(e)

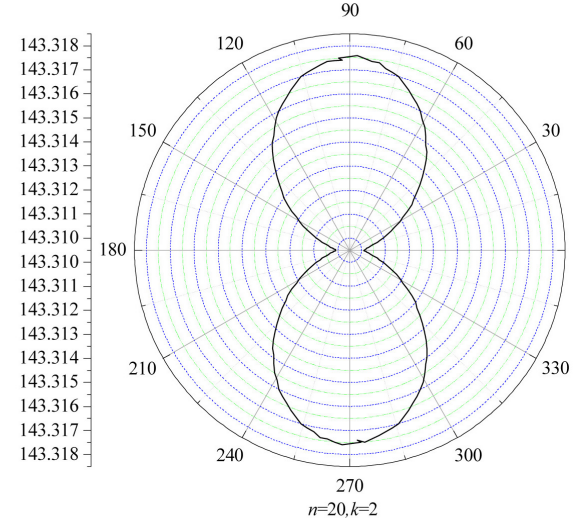

(b)

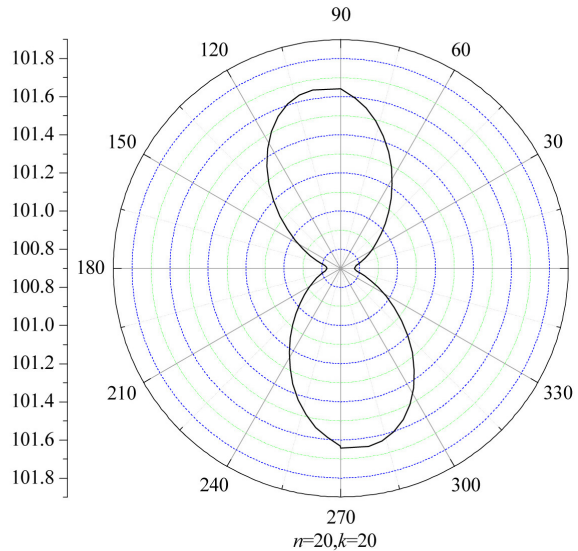

(d)

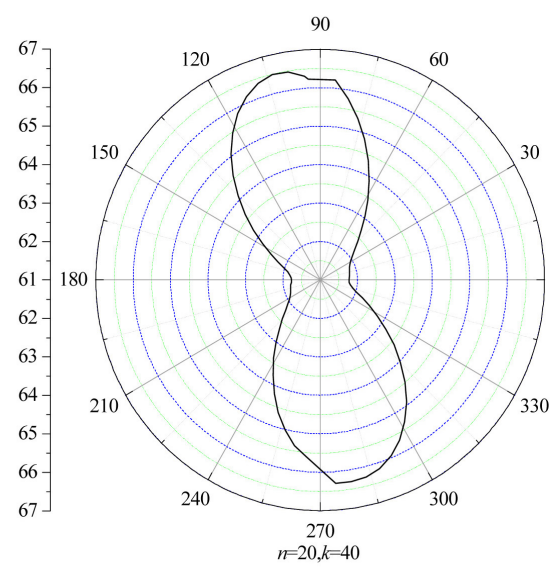

(f)

Figure 10. Phase velocity surface of shear wave in EMEP when $n=20, k=2,20$ and 40.

changes from 2 to 40 , this means that the closer the direction of wave propagating is the middle plane $x_{1} 0 x_{2}$, the more different the phase velocities of the wave propagating along the different directions are. It can be found from comparison of Figure 9(b), Figure 9(d), Figure 9(f) that the phase velocity surface rotates counter clockwise with the change of wave number $k$ from 2 to 40 , and the phase velocity plane is anti-symmetry about coordinate origin.

To further clarify the conclusions obtained from Figure 9, Figure 10 is drawn 
when the representative wave number $n=20$ and $k=2,20$ and 40. The same conclusions can be obtained from Figure 10 as that from Figure 9.

\section{Conclusions}

The bulk wave in the electromagnetic elastic plate is studied, and the following conclusions are obtained by the numerical examples:

1) The types of P-wave and S-wave reflected by the boundary of the plate remain unchanged.

2) The dispersion of the wave propagating along the mid-plane is less than that of the wave propagating along the plate thickness.

3) The closer the propagating direction of the longitudinal and the shear waves are to the middle plane, the more different their phase velocities in different directions are.

4) The phase velocity surface of the shear wave is anti-symmetry about the coordinate origin.

5) The phase velocity surface of a longitudinal wave is symmetrical about $\theta=75^{\circ}$ and $\theta=165^{\circ}$, this means that EMEP is approximately orthotropic.

\section{Acknowledgements}

This work is supported by National Natural Science Foundation of China under the Grant Number 11372109.

\section{Conflicts of Interest}

The authors declare no conflicts of interest regarding the publication of this paper.

\section{References}

[1] Harse, G., Dougherry, J.P. and Newnham, R.E. (1993) Theoretical Modelling of 3-0/0-3 Magntoelectric Composites. International Journal of Applied Electromagnetics in Materials, 4, 161-171.

[2] Avellaneda, M. and Harse, G. (1994) Magnetoelectric Effect in Piezoelectric Magnetostrictive (2-2) Composites. Journal of Intelligent Material Systems and Structure, 5, 501-513. https://doi.org/10.1177/1045389X9400500406

[3] Cupiał, P. (2015) Three-Dimensional Perturbation Solution of the Natural Vibrations of Piezoelectric Rectangular Plates. Journal of Sound and Vibration, 351, 143-160. https://doi.org/10.1016/j.jsv.2015.04.006

[4] Nie, G.Q., Liu, J.X., Fang, X.Q. and An, Z.J. (2012) Shear Horizontal (SH) Waves Propagating in Piezo-Electric-Piezomagnetic Bilayer System with an Imperfect Interface. Acta Mechanica, 223, 1999-2009.

https://doi.org/10.1007/s00707-012-0680-6

[5] Ezzin, H., Amor, M.B. and Ghozlen, M.H.B. (2017) Lamb Waves Propagation in Layered Piezoelectric/Piezomagnetic Plates. Ultrasonics, 76, 63-69. https://doi.org/10.1016/j.ultras.2016.12.016

[6] Guo, X., Wei, P.J., Li, L. and Lan, M. (2018) Effects of Functionally Graded Interlayers on Dispersion Relations of Shear Horizontal Waves in Layered Piezoelec- 
tric/Piezomagnetic Cylinders. Applied Mathematical Modelling, 55, 569-582. https://doi.org/10.1016/j.apm.2017.11.029

[7] Yu, J.G., Lefebvre, J.E. and Zhang Ch. (2014) Guided Wave in Multilayered Piezoelectric-Piezomagnetic Bars with Rectangular Cross-Sections. Composite Structures, 116, 336-345. https://doi.org/10.1016/j.compstruct.2014.04.025

[8] Pang, Y., Liu, Y.S., Liu, J.X., et al. (2016) Propagation of SH Waves in an Infinite/Semi-Infinite Piezoelectric/Piezomagnetic Periodically Layered Structure. UItrasonics, 67, 120-128. https://doi.org/10.1016/j.ultras.2016.01.007

[9] Wang, Y.Z., Li, F.M., Huang, W.H., et al. (2008) Wave Band Gaps in Two-Dimensional Piezoelectric/Piezomagnetic Phononic Crystals. International Journal of Solids and Structures, 45, 4203-4210.

https://doi.org/10.1016/j.ijsolstr.2008.03.001

[10] Li, L. and Wei, P.J. (2014) The Piezoelectric and Piezomagnetic Effect on the Surface Wave Velocity of Magneto-Electro-Elastic Solids. Journal of Sound and Vibration, 333, 2312-2326. https://doi.org/10.1016/j.jsv.2013.12.005

[11] Pang, Y., Wang, Y.S., Liu, J.X. and Fang, D.N. (2008) Reflection and Refraction of Plane Waves at the Interface Between Piezoelectric and Piezomagnetic Media. International Journal of Engineering Science, 46, 1098-1110.

https://doi.org/10.1016/j.ijengsci.2008.04.006

[12] Arfi, M. (2016) Analysis of Wave in a Functionally Graded Magneto-Electro-Elastic Nano-Rod Using Nonlocal Elasticity Model Subjected to Electric and Magnetic Potentials. Acta Mechanica, 227, 2529-2542.

https://doi.org/10.1007/s00707-016-1584-7 


\section{Appendix A}

$$
\begin{aligned}
& a_{11}=\left(C_{1122}-C_{1133}\right) k_{1} k_{2}+0.5 C_{1212} k_{1}^{2}, \\
& a_{12}=\left(-C_{1111}+C_{1133}\right) k_{1}^{2}-0.5 C_{1212} k_{1} k_{2}-\rho \omega^{2} \\
& a_{13}=-0.5 C_{3131} k_{1} k_{2} \text {, } \\
& a_{14}=-e_{111} k_{1} k_{2} \text {, } \\
& a_{15}=e_{111} k_{1}^{2} \text {, } \\
& a_{16}=-q_{111} k_{1} k_{2} \text {, } \\
& a_{17}=q_{111} k_{1}^{2} \\
& a_{21}=-0.5 C_{3131} k_{1}^{2} k_{2} \text {, } \\
& a_{22}=0.5 C_{3131} k_{1}^{3}-0.5 C_{3131} k_{1}\left(\eta_{m}^{\mathrm{s}}\right)^{2} \text {, } \\
& a_{23}=\left(C_{1111}+C_{1122}\right) k_{1}^{2} k_{2}+0.5 C_{1212} k_{1}^{3}+0.5 C_{1212} k_{1} k_{2}^{2}-\rho \omega^{2} k_{2} \text {, } \\
& a_{24}=e_{311} k_{1}^{2} k_{2} \text {, } \\
& a_{25}=-e_{311} k_{1}^{3} \\
& a_{26}=q_{311} k_{1}^{2} k_{2} \text {, } \\
& a_{27}=-q_{311} k_{1}^{3} \text {, } \\
& a_{31}=\left(C_{2222}-C_{2233}\right) k_{2}^{2}-0.5 C_{2121} k_{1} k_{2}-\rho \omega^{2} \\
& a_{32}=\left(-C_{2211}+C_{2233}\right) k_{1} k_{2}-0.5 C_{2121} k_{2}^{2} \text {, } \\
& a_{33}=-0.5 C_{2323} k_{1} k_{2} \text {, } \\
& a_{34}=e_{222} k_{2}^{2} \text {, } \\
& a_{35}=-e_{222} k_{1} k_{2} \text {, } \\
& a_{36}=q_{222} k_{2}^{2} \text {, } \\
& a_{37}=-q_{222} k_{1} k_{2} \text {, } \\
& a_{41}=-0.5 C_{2323} k_{2}^{3}+0.5 C_{2323} k_{2}\left(\eta_{m}^{s}\right)^{2} \text {, } \\
& a_{42}=0.5 C_{2323} k_{1} k_{2}^{2} \text {, } \\
& a_{43}=\left(C_{2211}-C_{2222}\right) k_{1} k_{2}^{2}+0.5 C_{2121}\left(k_{2} k_{1}^{2}+k_{2}^{3}\right)-\rho \omega^{2} k_{1} \text {, } \\
& a_{44}=e_{232} k_{2}^{3} \text {, } \\
& a_{45}=-e_{232} k_{1} k_{2}^{2} \\
& a_{46}=q_{232} k_{2}^{3} \text {, } \\
& a_{47}=-q_{232} k_{1} k_{2}^{2} \text {, } \\
& a_{51}=\left(C_{3322}+C_{3333}\right) k_{2}\left(\eta_{m}^{\mathrm{s}}\right)^{2}-\rho \omega^{2} k_{2} \text {, } \\
& a_{52}=\left(C_{3311}-C_{3333}\right) k_{1}\left(\eta_{m}^{\mathrm{s}}\right)^{2}+\rho \omega^{2} k_{1} \text {, } \\
& a_{53}=0.5\left(C_{3131} k_{2}-C_{2323} k_{1}\right)\left(\eta_{m}^{\mathrm{s}}\right)^{2} \text {, } \\
& a_{54}=e_{333} k_{2}\left(\eta_{m}^{\mathrm{s}}\right)^{2} \text {, } \\
& a_{55}=-e_{333} k_{1}\left(\eta_{m}^{\mathrm{s}}\right)^{2} \text {, } \\
& a_{56}=q_{333} k_{2}\left(\eta_{m}^{\mathrm{s}}\right)^{2} \text {, } \\
& a_{57}=-q_{333} k_{1}\left(\eta_{m}^{\mathrm{s}}\right)^{2} \text {, } \\
& a_{61}=0.5 e_{311} k_{1}^{2} k_{2}+0.5 e_{232} k_{2}\left[k_{2}^{2}+\left(\eta_{m}^{s}\right)^{2}\right]+\left(e_{222}+e_{333}\right) k_{2}\left(\eta_{m}^{\mathrm{s}}\right)^{2} \text {, } \\
& a_{62}=0.5 e_{311} k_{1}\left[k_{1}^{2}+\left(\eta_{m}^{\mathrm{s}}\right)^{2}\right]-0.5 e_{232} k_{1} k_{2}^{2}+\left(e_{111}-e_{333}\right) k_{1}\left(\eta_{m}^{\mathrm{s}}\right)^{2} \text {, } \\
& a_{63}=0 \text {, } \\
& a_{64}=g_{111} k_{1}^{2} k_{2}+g_{222} k_{2}^{3}+g_{333} k_{2}\left(\eta_{m}^{s}\right)^{2} \text {, }
\end{aligned}
$$




$$
\begin{aligned}
& a_{65}=-g_{111} k_{1}^{3}-g_{222} k_{1} k_{2}^{2}-g_{333} k_{1}\left(\eta_{m}^{\mathrm{s}}\right)^{2}, \\
& a_{66}=a_{67}=0 \\
& a_{71}=0.5 q_{311} k_{1}^{2} k_{2}+0.5 q_{232} k_{2}\left[k_{2}^{2}+\left(\eta_{m}^{\mathrm{s}}\right)^{2}\right]+\left(q_{222}+q_{333}\right) k_{2}\left(\eta_{m}^{\mathrm{s}}\right)^{2} \\
& a_{72}=0.5 q_{311} k_{1}\left[k_{1}^{2}+\left(\eta_{m}^{\mathrm{s}}\right)^{2}\right]-0.5 q_{232} k_{1} k_{2}^{2}+\left(q_{111}-q_{333}\right) k_{1}\left(\eta_{m}^{\mathrm{s}}\right)^{2}, \\
& a_{73}=a_{74}=a_{75}=0, \\
& a_{76}=\mu_{111} k_{1}^{2} k_{2}+\mu_{222} k_{2}^{3}+\mu_{333} k_{2}\left(\eta_{m}^{\mathrm{s}}\right)^{2}, \\
& a_{77}=-\mu_{111} k_{1}^{3}-\mu_{222} k_{1} k_{2}^{2}-\mu_{333} k_{1}\left(\eta_{m}^{\mathrm{s}}\right)^{2}, \\
& a_{81}=0.5 C_{3131} k_{1} k_{2}+0.5 C_{2323}\left[k_{2}^{2}-\left(\eta_{m}^{\mathrm{s}}\right)^{2}\right], \\
& a_{82}=-0.5 C_{2323} k_{1} k_{2}-0.5 C_{3131}\left[k_{1}^{2}-\left(\eta_{m}^{\mathrm{s}}\right)^{2}\right] \\
& a_{83}=-\left(C_{3311}+C_{3322}\right) k_{1} k_{2}, \\
& a_{84}=-e_{311} k_{1} k_{2}, \\
& a_{85}=e_{311} k_{1}^{2}, \\
& a_{86}=-q_{311} k_{1} k_{2}, \\
& a_{87}=q_{311} k_{1}^{2}
\end{aligned}
$$

\section{Appendix B}

$$
\begin{aligned}
& b_{11}=C_{1111} k_{1}^{2}+C_{1122} k_{2}^{2}+C_{1133}\left(\eta_{m}^{\mathrm{d}}\right)^{2}+C_{1212} k_{1} k_{2} \\
& b_{12}=e_{111}\left(\eta_{m}^{\mathrm{d}}\right)^{2} \\
& b_{13}=q_{111}\left(\eta_{m}^{\mathrm{d}}\right)^{2} \\
& b_{21}=C_{2211} k_{1}^{2}+C_{2222} k_{2}^{2}+C_{2233}\left(\eta_{m}^{\mathrm{d}}\right)^{2}+C_{2121} k_{1} k_{2} \\
& b_{22}=e_{222}\left(\eta_{m}^{\mathrm{d}}\right)^{2} \\
& b_{23}=q_{222}\left(\eta_{m}^{\mathrm{d}}\right)^{2} \\
& b_{31}=C_{3311} k_{1}^{2}+C_{3322} k_{2}^{2}+C_{3333}\left(\eta_{m}^{\mathrm{d}}\right)^{2} \\
& b_{32}=e_{333}\left(\eta_{m}^{\mathrm{d}}\right)^{2} \\
& b_{33}=q_{333}\left(\eta_{m}^{\mathrm{d}}\right)^{2}
\end{aligned}
$$

